\title{
Tuberculosis in England and Wales in the next 20 years
}

\author{
J. R. Bignall \\ Brompton Hospital, London, S.W.3
}

\begin{abstract}
Summary
Present trends in the incidence of tuberculosis are reviewed.

Extrapolation suggests that there will only be about 1200 notifications in 1980 . By 1990 it is probable that the disease will be no commoner than non-tuberculous meningitis is now, and by 2010 it should be of interest to the medical historian only.
\end{abstract}

Tuberculosis is already an uncommon disease. The notification rate for respiratory tuberculosis in England and Wales in 1970 was about twenty per 100,000 . Thus a general practitioner with a list of 3500 could expect to see only one new case every 18 months. A hospital serving a population of 300,000 would have sixty new cases a year. If there were no special chest department and five general physicians, each might see one new patient a month.

There are, of course, considerable regional differences. In 1968, for instance, the rate for the whole country for males was thirty per 100,000 ; but for the conurbations it was forty-three. The corresponding rates in females were fourteen and twenty-one. The rates in the rural areas were much lower: in men fifteen and in women only seven.

To plan rationally the measures required to eradicate tuberculosis it is necessary to have some estimate of the rate of decline of the disease with present control procedures. On this can be based future policy.

To attempt to forecast as far ahead as 1990 may appear folly. But it probably isn't. In spite of the well-known inaccuracies of notification, the rates can in this country give useful information of trends. Notification rates have been falling exponentially. When plotted on semi-log paper the rates during the past 12 years come quite close to a straight line (Fig. 1).

The annual decrease in total respiratory notifications is at present about $9 \frac{1}{2} \%$. For males it is $9 \%$ and females about $10 \frac{1}{2} \%$.

The rates are different in different age groups. In those aged 65 years or older the annual decrease has been only $5-6 \%$, whereas in the 15-24 year age group the decrease in males has been about $11 \%$ a year and in females as high as $15 \%$.

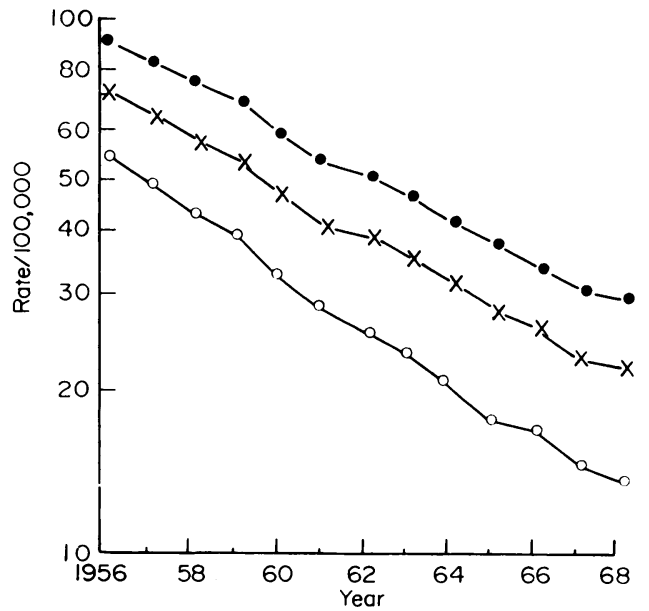

FIG. 1. Notification rates for pulmonary tuberculosis per 100,000 in the period $1956-68$. -0 , male; $O_{-} \mathrm{O}$, female; $\times-\times$, persons.

In some age groups there is a suggestion of a change in the rate of decline during the past 10 years (Fig. 2). In males there was a decrease in annual rate of decline around 1962 in those aged 15-34. In females the decrease in this age group began around 1966. In males aged 15-24 the annual rate of decline changed from 17 to $10 \%$. In males aged $25-34$ the rate of decline was not altered; but the notification rate did not fall between 1961 and 1962, remaining at sixty per 100,000 . Thereafter it continued to decrease at the rate of about $13 \%$ per year. Similar changes occurred in females from 1966. In those aged 15-24 the annual rate of decline changed from 17 to $8 \%$ and in those aged $25-34$ from 13 to $7 \%$.

These changes may have been caused by immigration.

The rate of decline varies not only with age, but also with the density of population (Fig. 3). During the period 1965-68 there was about a $15 \%$ decrease of the rate in conurbations and urban areas with populations of 100,000 or more. But in rural areas the rate decreased by $35 \%$.

The period for which the rates are available1965-68-is short; but there is a suggestion of certain trends that also could be associated with 


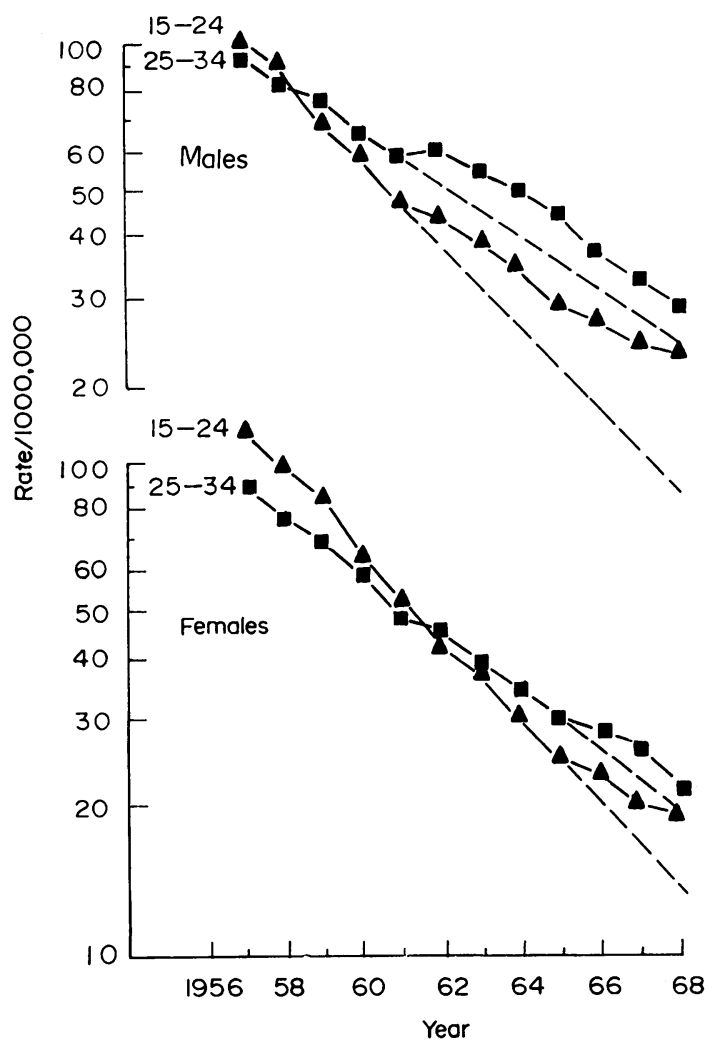

FIG. 2. Notification rates for pulmonary tuberculosis per 100,000 in the period $1956-68$ in males and females, aged 15-24 years and 25-34 years.

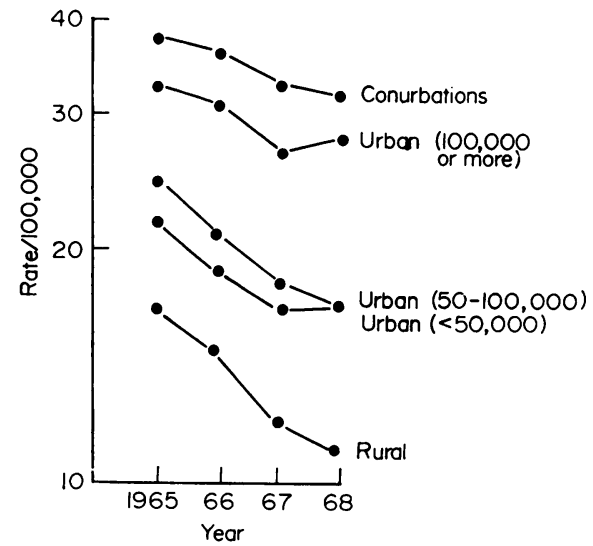

Fig. 3. Notification rates for pulmonary tuberculosis per 100,000 in the period $1965-68$ related to the place of notification. immigration. Thus, in both males and females there응 was little difference between conurbations and rural⿳亠丷⿵冂丶 areas in the changes in persons aged 45 years or 3 older. For instance, the rate in men of 45-64 fell by@ $24 \%$ in conurbations and $23 \%$ in rural areas; the $C$ corresponding proportions for women were 17 and $\vec{F}$ : $20 \%$. However, in both men and women the fall was $\stackrel{5}{?}$ much less in conurbations than rural areas in all age? groups below 45 years. Since there are probably $\frac{\bar{\sigma}}{\bar{c}}$ fewer immigrants in rural areas, and few anywhere $\vec{\odot}$ over the age of 45 , these changes could have been $\triangle$ contributed to by the known high rates in certain groups of immigrants, though it is unlikely that $\vec{\circ}$ there were no other factors.

It seems likely that the general decline will con- $\vec{\omega}$ tinue at approximately the same rate. Reduction ing the rate of decline could occur through further large scale immigration, war or a prolonged period of severe economic depression. All seem unlikely. In- $-\vec{t}$ creasing concentration of the population into small $\mathrm{G}$ areas-conurbations-could reduce the rate. The $\omega$ rate of decline might on the other hand be increased of by decreasing the risk of infection or reducing the risk of breakdown among those already infected. This might be contributed to by increased economic $\vec{z}$ prosperity and 'secondary chemoprophylaxis'. The을 former, unfortunately, seems unlikely to be a major factor in the near future; and the second is doub fully practicable or necessary, except in specigl groups.

If the rate of decline continues at around $10 \%$ p annum, the notification rate in 1980 will be six per 100,000 and in 1990 two per 100,000 -four in males and one-and-a-quarter in females (Fig. 4). The rate

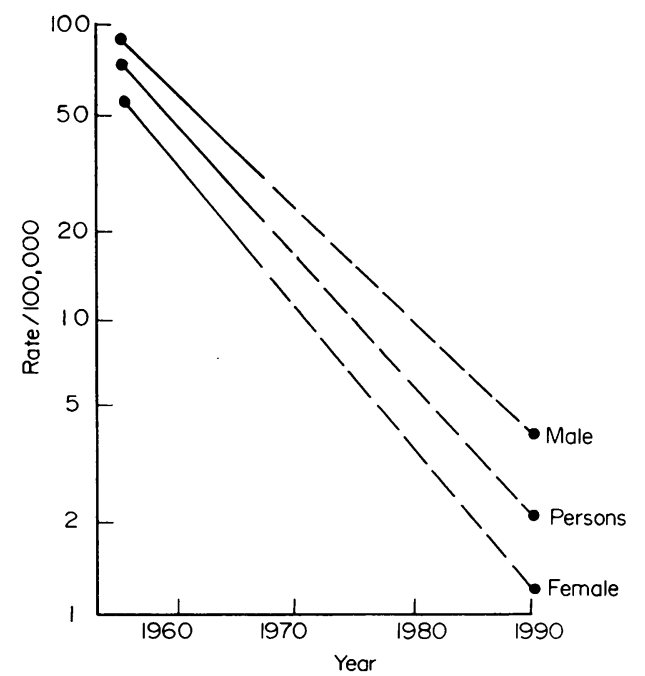

Fig. 4. Projected notification rates for pulmonary tuberculosis per 100,000 to the year 1990 , assuming a constant rate of decrease. 
in both sexes in the 5-14 age group (which has the smallest rates of all groups) will be only about one per 100,000 ; and the highest rate-in men over 64 will be about thirteen. With the estimated population of the United Kingdom at 62 million, there will be only about 1200 notifications.

To form an idea of how rare tuberculosis will be, these projected rates for 20 years ahead can be compared with the present rates for some rare diseases (Table 1). Thus, tuberculosis will in 1990 be as rare as acute, non-tuberculous meningitis at present. It will be only ten times more common than is acute encephalitis and typhoid fever. It will be about as common, as is at present, death from carcinoma of the larynx or gall bladder, malignant hypertension, suicide by hanging and accidental burns.

TABLE 1. Estimated notification rate per 100,000 for pulmonary tuberculosis in 1990 related to some notification and death rates in 1968

Rate $/ 100,000$

$\begin{array}{ll}1990 & \\ \text { Pulmonary tuberculosis } & 2 \cdot 0 \\ 1968 & \\ \text { Notifications: } & 1 \cdot 6 \\ \text { Acute meningitis } & 0 \cdot 2 \\ \text { Acute encephalitis } & 0 \cdot 2 \\ \text { Typhoid fever } & \\ \text { Deaths: } & 2 \cdot 3 \\ \text { Carcinoma larynx } & 1 \cdot 4 \\ \text { Malignant hypertension } & 1 \cdot 6 \\ \text { Accidents-burns } & 1 \cdot 2 \\ \text { Suicide-hanging } & \end{array}$

The rates and the speed of decline will probably still vary in different parts of the country. For conurbations the rate may be as high as seven per 100,000 , for rural areas as low as $\mathbf{0} \cdot \mathbf{8}$. A conurbation of 10 million people may have 700 notifications in 1990. A general hospital serving a town of 300,000 inhabitants will have about fifteen new cases in that year. Assuming that each patient will be kept under observation for 5 years (which may by then be unnecessary), there will be seventy-five patients on the register. If on average four contacts are examined for each new case, only sixty will need to be examined during the year. A general practitioner in a rural area with a list of 3000 may see only one new case during 40 years of medical practice.

A disease of such rarity will not require a special organization to deal with it. The fifteen new cases in the population of 300,000 can be diagnosed by the general physicians and treated by them and by the general practitioners. Contact examinations can be done by the community health department.

It will be unnecessary for there to be any register of cases other than a national one. Once the name and certain other particulars about the newly diagnosed patient have been notified to the national register, no further administrative action will be required from the physician. It will be done by the computer. Indeed, such a system could be used now. The number of new notifications and the numbers on the clinic registers are certainly not so great that a computer could not deal with them.

Diagnosis of pulmonary tuberculosis in most cases requires little skill. It needs some experience of interpreting chest radiographs and knowledge of the necessity for routine bacteriological examination of the sputum of any patient with abnormal lung fields. Specialists are not required. Treatment is in most cases easy for the physician, though not for the patient. By 1990 it is likely that shorter periods of chemotherapy will be used, further simplifying the control of treatment. Any reasonably competent physician or general practitioner will be able to treat the patients. Specialists will not be required.

No doubt, with such a rare disease, mistakes will be made in both diagnosis and treatment. However, paradoxically, it may be that relatively fewer will be made when tuberculosis becomes a disease to be recognized and treated by any general physician, rather than being looked upon as wholly the concern of the specialist. It will probably not be forgotten as frequently as some might fear. Typhoid fever is at present rare-only 0.2 per 100,000 . But it is still remembered in the differential diagnosis of 'P.U.O.' by every medical student.

Comments on the decline of tuberculosis are often followed by exhortations about complacency. It is unwise to be complacent. But it is also unwise to be unrealistic. Tuberculosis is declining rapidly; and I suspect that the decline will continue whatever programme of control is adopted and whatever imperfections there are in it. Some factors might slightly speed up the decline, others slightly retard it. The investigations of the Tuberculosis Surveillance Research Unit of the International Union Against Tuberculosis have already given valuable information on the decline in infection rate and its independence of economic factors and on the failure of practicable population screening by radiography to reduce sources of infection. Their further investigations may indicate where the maximum efforts should be made and where no further efforts are required.

In this country mass radiography of populations is rightly being discontinued; and mass BCG vaccination will certainly be discontinued during the next two decades. Mass methods are giving way to selective procedures.

As chest clinics deal less and less with tuberculosis and newly appointed chest physicians know less and less about it, it might at some stage be profitable to move all preventive measures back to the public 
health departments. It would then be their direct responsibility to investigate sources of infection and to circumscribe local outbreaks - as is now done with other : infectious diseases. Diagnosis and treatment would remain with the clinicians, but they would no longer be concerned with those aspects of tuberculosis control for which they have little interest.

I think it likely that by 1990 the few remaining patients with tuberculosis will be diagnosed and treated by general physicians and practitioners as they now diagnose and treat other infectious diseases. The public health departments will be concerned with all aspects of prevention. A few regional and national tuberculosis advisers may still be required.

The Tuberculosis Officer disappeared 20 years ago.
In another 20 years the chest physician as the centrafo figure of tuberculosis control will also have dis-气 appeared. And by 2010 tuberculosis itself-with an $\frac{3}{3}$ incidence of 0.2 per 100,000 -will be of no more im- $\stackrel{\AA}{\varrho}$ portance to the community than typhoid fever is $ᄃ$ today. It will be of interest only to the medical $\vec{F}$ historian.

\section{Addendum}

Since this report was prepared, the notification $\frac{\bar{\omega}}{\vec{D}}$ rates for 1969 have become available. The general trends are unchanged. The rate per 100,000 fell to twenty from twenty-two in 1968 (a $9 \%$ decrease); $\overrightarrow{0}$ the rate for males fell from thirty to twenty-seven and for females fourteen to thirteen. 